\title{
Planar Force-Constant Method for Lattice Dynamics of Cubic III-Nitrides
}

\author{
H. W. Leite Alves, J. L. A. Alves, \\ Departamento de Ciências Naturais, FUNREI, \\ C.P.: 110, CEP: 36300-000, São João del Rei, MG, Brazil \\ L. M. R. Scolfaro, and J. R. Leite \\ LNMS, Departamento de Física dos Materiais e Mecânica, USP \\ C.P.: 66.318, CEP: 05389-970, São Paulo, SP, Brazil
}

Received on 23 April, 2001

\begin{abstract}
Using the density-functional theory within the Full Potential Linear Augmented PlaneWave (FP-LAPW) method, we have calculated, in this work, the equation of state, the effective charges and the phonon dispersion along [100] and [111] directions for the cubic III-Nitrides(c-BN, c-AlN, c-GaN and c-InN), including hydrostatic strains dependence. A good agreement with the micro-Raman scattering experiment is obtained for the phonon modes at $\Gamma$. Our results show that, for c-InN case, the apparent discrepancy between the known experimental results is a consequence of hydrostatic effects on the sample due to differences of the used Raman methods.
\end{abstract}

\section{Introduction}

The group III-nitrides(AlN, GaN, InN) and the corresponding alloys have attracted great interest due to their successful applications in the electronic and optoelectronic device technology [1]. Unfortunately, the amount of theoretical knowledge on cubic IIINitrides(c-IIIN) is limited to a handful of works [2$8]$ and, particularly, the experimental data on phonon spectra and vibrational properties are rather scarce: only Raman scattering measurements were reported [911], which deals with the zone-center modes. So, the knowledge of the whole spectra remains restricted, principally, to the theoretical calculations.

Due to the lack of lattice-matched substrates, the c-IIIN samples are affected by the high density of defects and strain present in the films and it is therefore unclear how well these data represent the true bulk values. In the c-InN case, for instance, it is interesting to note that, despite the fact that only two different Raman spectra $[10,11]$ were reported, both were recorded on the same sample and their results agree only for the longitudinal mode at $\Gamma$. Following Ref. 11, the supposed wrong assignment for the $\mathrm{TO}(\Gamma)$ mode is probably due to the similarity of the nonresonant spectra of Ref. 10 to the second-order spectra of InAs.

In the present work, we report our preliminary results for the lattice dynamics of c-IIIN, including hydro- static strains, to supply the missing information on the vibrational properties of these compounds. The phonon frequencies at [100] and [111] directions were obtained within the planar force-constant model $[12,13]$ : it consists in selecting one direction of the wavevector $\vec{k}$ and in representing the lattice vibrations as the motion of rigid planes of atoms perpendicular to $\vec{k}$ and connected by inter-planar forces. In order to determine these force constants, we have done supercell calculations using the density functional theory within the local density approximation and the full-potential linear augmented-plane-wave method(FP-LAPW) [14]. For the electron-gas exchange-correlation term, we have used the Ceperley-Alder data [15]. In the FP-LAPW method, the muffin-tin radii used were 1.90 a.u.(for $\mathrm{InN}), 1.70$ a.u.(for $\mathrm{GaN}), 1.55$ a.u.(for $\mathrm{AlN}$ ), and 1.29 a.u.(for $\mathrm{BN}$ ) and the self-consistent total energy was converged to 10angular momentum was $\ell=10$ for the wave function inside the spheres and $\ell=4$ for the computational of non-muffin-tin elements. The Ga-3d and In-4d electron states were treated explicity as part of the valence band states in order to take into account the hybridizations between Ga-3d, In-4d and N-2s states. The forces on atoms in the out-of-equilibrium situations are calculated using the Hellmann-Feynman theorem [16]. 


\section{Equilibrium Properties}

Before to proceed the supercell calculations, we have evaluated the total energy for various values of the lattice constant in order to have a good description of the equilibrium properties for c-IIIN. The results were fitted by the Murnaghan equation of state. We have, then, obtained $\mathrm{a}_{0}=3.585(\mathrm{c}-\mathrm{BN}), 4.346(\mathrm{c}-$ AlN), 4.465(c-GaN), 4.976(c-InN) $\AA, \mathrm{B}_{0}=4.01(\mathrm{c}-\mathrm{BN})$, 2.11(c-AlN), 2.02(c-GaN), 1.48(c-InN) Mbar and $\mathrm{B}_{0}{ }_{0}=$ $3.5(\mathrm{c}-\mathrm{BN}), 3.7(\mathrm{c}-\mathrm{AlN}), 5.5(\mathrm{c}-\mathrm{GaN}), 4.1(\mathrm{c}-\mathrm{InN})$. Our results for the lattice constant, Bulk modulus and its pressure derivative are in good agreement with the available experimental results(within $\sim 5 \%$ ) $[2,10]$, as well as with previous calculations [3-8].

\section{Planar Force Constants}

In order to determine the force constants ab initio, we proceed in the same way as described in Ref. 12 and Ref. 13. To describe the phonon dispersion at the [100] direction, we used supercells which were four and six times the volume of the elementary cell of c-IIIN, containing eight and twelve atoms, respectively. For the [111] direction, in the similar way, we have used supercells which was three and six times the elementary cell of c-IIIN, containing, then, six and twelve atoms.

To obtain the force constants which describe the phonon dispersion along $\Gamma$ to $\mathrm{X}$ direction, we displace an entire plane of atoms(plane $n=0)$ in the [100] direction(for the longitudinal modes(LM)) or in the [011] direction(for the transversal modes(TM)). For $\Gamma$ to L direction, with the [111] supercell, such displacement was in the [111] direction for the LM and in the [11-2] direction for the TM. To avoid anharmonicity effects, we have done five displacements $(0, \pm \mathrm{u}, \pm 2 \mathrm{u})$ in each planar force calculation for c-IIIN at the theoretical equilibrium lattice constant, as described in the Ref. 17.

The obtained phonon frequencies are summarized in Table I, compared with the experimental data obtained by Raman measurements. The overall agreement is $\operatorname{good}(\sim 10 \%)$. With relation to others calculations $[7,8]$, our obtained results also compare well. Other parameters and further details of our calculations, including the convergence with the sampling of the Brillouin zone[18], will come up soon in another publication.

\section{Hydrostatic Strains}

In Fig. 1, the calculated dependence of the $\mathrm{TO}(\Gamma)$ mode with the applied hydrostatic strains is shown for the $\mathrm{c}-\mathrm{InN}$ case. Here, we have considered only out- ward strains because it is directly related to the experimental growth conditions of the used sample[10]: a thin film of $\mathrm{c}-\operatorname{InN}\left(\mathrm{a}_{0}=4.98 \AA\right)$ deposited over a buffer layer of $\operatorname{InAs}\left(\mathrm{a}_{0}=6.06 \AA\right)$, which is over a substrate of $\operatorname{GaAs}(001)\left(\mathrm{a}_{0}=5.65 \AA\right)$. Indeed, in these conditions, the lattice mismatch between the film and the substrate(plus a buffer layer) induces outward strains on c-InN. Moreover, we have chosen hydrostatic strains once that there is no experimental report about induced uniaxial and/or biaxial strains during the thin film growth. Also, we indicated, on the figure, both Raman results for this particular mode. It is clear, from the figure, that the frequency of the $\mathrm{TO}(\Gamma)$ mode is dramatically changed with small hydrostatic strain variation. Such variation, in our preliminary results, showed to be linear, which should be reasonable for applied small strains [19]. However, this picture was not observed for the $\mathrm{LO}(\Gamma)$ mode, which remained almost insensitive to small strain variations in our simulations.

Because of the nature of the $\mathrm{TO}(\Gamma)$ mode with applied strains depicted in Fig. 1, it becomes clear that there is no controversy among the observed experimental results. In Ref. 10, the authors have used microRaman to determine the $\mathrm{TO}(\Gamma)$ frequency. If the sample have any small imperfection on the scanned area, even preserving its overall symmetry(as small hydrostatic deviations like that of Fig. 1), large deviations will be detected on the final value for the $\mathrm{TO}(\Gamma)$ frequency, leading to possible wrong assignment for that. We note that, between the both Raman results, we can expect strains at order of $0.5 \%$ only. So, we can conclude that both experiments have detected the same mode - the apparent discrepancy is due to differences between the used Raman methods.

From our simulations of applied hydrostatic strains, the evaluated Grüneisen parameters $\gamma$ for the $\mathrm{TO}(\Gamma)$, $\mathrm{TO}(\mathrm{X})$ and $\mathrm{TA}(\mathrm{X})$ modes were $1.8,1.7$ and 1.2 , respectively. The higher values of $\gamma$ for the optical modes reflect that this band is flat along [100] direction, as observed in other calculations [9]. Also, as observed in our previous calculations for BP and for BN [20], the $\mathrm{TA}(\mathrm{X})$ is a soft mode and its frequency decreases with increasing pressure: its Grüneisen parameter $\gamma$ has a negative value. Complete description of our obtained results for applied strains on the dynamical properties of c-InN, as well as for the other III-Nitrides, will be published soon in another publication. 
Table I. Summary of the calculated phonon frequencies(in $\left.\mathrm{cm}^{-1}\right)$ for c-IIIN. In the parenthesis are the experimental data, extracted from Refs. 7-12.

\begin{tabular}{lcccccccccc}
\hline \hline & $\mathrm{LO}(\Gamma)$ & $\mathrm{TO}(\mathrm{\Gamma})$ & $\mathrm{LO}(\mathrm{X})$ & $\mathrm{LA}(\mathrm{X})$ & $\mathrm{TO}(\mathrm{X})$ & $\mathrm{TA}(\mathrm{X})$ & $\mathrm{LO}(\mathrm{L})$ & $\mathrm{LA}(\mathrm{L})$ & $\mathrm{TO}(\mathrm{L})$ & $\mathrm{TA}(\mathrm{L})$ \\
\hline $\mathrm{BN}$ & $1153.1(1305)$ & $1057.7(1055)$ & 1075.5 & 940.1 & 810.7 & 799.9 & 1026.3 & 929.3 & 727.4 & 666.8 \\
$\mathrm{AlN}$ & $769.2(902)$ & $665.6(655)$ & 689.0 & 579.4 & 610.8 & 422.3 & 573.1 & 497.2 & 499.1 & 358.6 \\
$\mathrm{GaN}$ & $778.0(741)$ & $566.4(555)$ & 739.0 & 385.2 & 595.7 & 248.1 & 614.2 & 309.3 & 490.1 & 212.3 \\
$\mathrm{InN}$ & $526.0(587)$ & $478.6(470)$ & 555.4 & 203.8 & 502.2 & 147.0 & 531.7 & 217.1 & 371.0 & 128.8 \\
\hline \hline
\end{tabular}

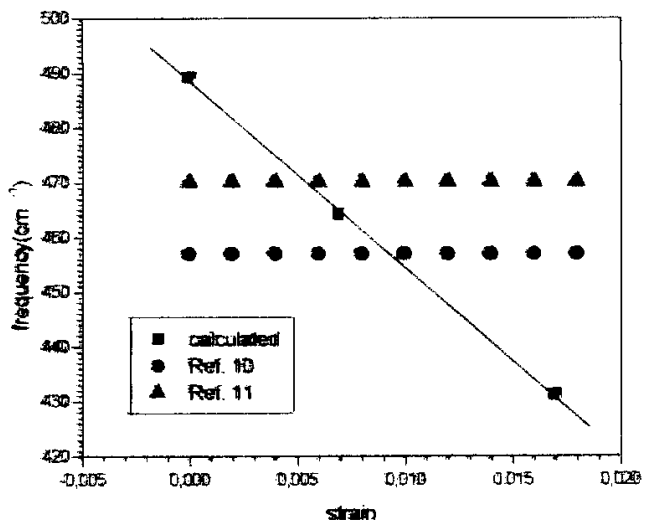

Figure 1. Calculated dependence of the $\mathrm{TO}(\Gamma)$ mode with the applied hydrostatic strains, compared with the experimental results from Ref. 10 and Ref. 11.

In summary, we have described our preliminary results for the lattice dynamics of c-IIIN using the Density Functional Theory, within the framework of FPLAPW. This procedure describes correctly the undistorted structure of c-IIIN in static equilibrium, and our results for lattice constant and Bulk modulus are in good agreement with the available experimental results. Having a good description of the static equilibrium of c-IIIN, we have calculated its phonon frequencies for the [100] and [111] directions of the Brillouin zone(BZ) by using the Planar Force-constants Method. At the $\Gamma$ point of the BZ, our results agree well with those obtained by Raman Scattering. Our strain simulations reveal that the apparent divergence between the known experimental results is a consequence of hydrostatic effects on the sample due to differences of the used Raman methods. We hope that our calculations work as a guide for further experiments on this issue.

\section{Acknowledgements}

This work was partially supported by $\mathrm{CNPq}$ and by FAPEMIG.

\section{References}

1. S. C. Jain, M. Willander, J. Narayan and R. van Overstraeten, J. Appl. Phys. 87, 965(2000).
2. J. H. Edgar, Properties of group III Nitrides (INSPEC, London, 1994).

3. C. -Y. Yeh, Z. W. Lu, S. Froyen and A. Zunger, Phys. Rev. B46, 10086(1992).

4. A. F. Wright and J. S. Nelson, Phys. Rev. B51, 7866(1995).

5. M. van Schilfgaarde, A. Sher and A. -B. Chen, J. Cryst. Growth 178, 8(1997).

6. K. Kim, W. R. Lambrecht and B. Segall, Phys. Rev. B53, 16310(1996).

7. F. Bechstedt, U. Grossner and J. Furthmüller, Phys. Rev. B62, 8003(2000).

8. C. Bungaro, K. Rapcewicz and J. Bernholc, Phys. Rev. B61, 6720(2000).

9. H. Siegle, G. Kaczmarczyc, L. Filippidis, A. P. Litvinchuk, A. Hoffmann and C. Thomsen, Phys. Rev. B55, 7000(1997).

10. A. Tabata, A. P. Lima, L. K. Teles, L. M. R. Scolfaro, J. R. Leite, V. Lemos, B. Schöttker, T. Frey, D. Schikora and K. Lischka, Appl. Phys. Lett. 74, 362(1999).

11. G. Kaczmarczyk, A. Kaschner, S. Reich, A. Hoffmann, C. Thomsen, D. J. As, A. P. Lima, D. Schikora, K. Lischka, R. Averbeck and H. Riechert, Appl. Phys. Lett. 76, 2122(2000).

12. K. Kunc, Eletronic Structure, Dynamics and Quantum Structural Properties of Condensed Matter, edited by J. T. Devreese and P. E. Van Camp(Plenum Press, New York, 1985) p. 227.

13. G. P. Srivastava and K. Kunc, J. Phys. C: Solid State Phys. 21, 5087(1988).

14. P. Blaha, K. Schwarz, and J. Luitz, Wien97 - A Full Potential Linearized Augmented Plane Wave Package for Calculating Crystal Properties(Technical University of Vienna, 1997).

15. D. M. Ceperley and B. I. Alder, Phys. Rev. Lett. 45, 566(1980); J. Perdew and A. Zunger, Phys. Rev. B23, 5048(1981).

16. K. Kunc and R. M. Martin, Ab initio Calculation of Phonon Spectra, edited by J. T. Devreese, V. E. Van Doren and P. E. Van Camp(Plenum Press, New York, 1983) p. 65, and references therein.

17. S. Wei and M. Y. Chou, Phys. Rev. B50, 2221(1994).

18. The FP-LAPW method(Wien97 code) uses the improved tetrahedron method(P. E. Blöchl, O. 
Jepsen and O. K. Andersen, Phys. Rev. B49, 16223(1994)) to perform integrations over the Brillouin zone. In the tetrahedron method, the reciprocal space is divided into tetrahedra, within which matrix elements and band energies are linearized in $\vec{k}$. The new improved method maps identically onto the special-points in the scheme of Monkhorst and Pack(Phys. Rev. B13, 5188 (1976)) to avoid an evaluation of large number of tetrahedra. With this, the integration error decays exponentially with the distance between $\vec{k}$ points. Then, for a right accuracy of our obtained results, it is necessary to obtain their convergence with the number of $\vec{k}$ points which is related, in this method, with the number of tetrahedra.

19. E. M. Anastassakis, Morphic Effects in Lattice Dynamics, Dynamical Properties of Solids, edited by G. K. Horton and A. A. Maradudin(NorthHolland, Amsterdam, 1980), vol. 4, p. 157.

20. H. W. Leite Alves and K. Kunc, J. Phys.: Condens. Matter 4, 6603(1992); H. W. Leite Alves, J. L. A. Alves, J. L. P. Castineira and J. R. Leite, Mater. Sci. Eng. B59, 264(1999). 